\title{
XSPECT: A TELESCOPE/SPECTROMETER SYSTEM ON SPECTRUM RÖNTGEN GAMMA
}

\author{
Herbert W. Schnopper
}

Danish Space Research Institute, DK-2800 Lyngby, Denmark

\begin{abstract}
The SPECTRUM RONTGEN-GAMMA mission is being developed by the Babakin Center (BC) together with the Space Research Institute (IKI) of the Academy of Sciences, USSR and is scheduled for launch in 1993. Mission objectives include broad and narrow band imaging spectroscopy over a wide range of energies from the EUV through gamma rays with particular emphasis on the study of extragalactic objects. The Danish Space Research Institute (DSRI) BC and IKI share the responsibility for the preparation of the XSPECT system. Two thin foil telescopes which are conical shell approximations to Wolter 1 geometry, each with an aperture of $60 \mathrm{~cm}$ and a focal length of $8 \mathrm{~m}$, are designed to have a half-power width of less than 2 arcmin and will have collecting areas of 1700 and $1200 \mathrm{~cm}$ at 2 and $8 \mathrm{keV}$, respectively. Images and spectra will be recorded with position sensitive proportional counters with good spectral resolution. An objective Bragg crystal panel, placed in front of one of the telescopes, will make high resolution spectroscopic studies $\left(E / \Delta E \sim 10^{3}\right)$ of point- and extended sources. Other instruments are under consideration.
\end{abstract}

\section{INTRODUCTION}

SPECTRUM RONTGEN-GAMMA is expected to be the first of a new series of astronomical missions announced by the Academy of Sciences of the USSR. The payload under study (see Figure 1) consists of instruments intended for a broad range of astrophysical studies but with particular emphasis on extragalactic objects. Concentrating telescopes will be provided for EUV (EUVITA) and X-ray (JET-X and XSPECT) studies and a coded aperture mask telescope will image hard $X$-rays and soft gamma rays (MART). Supplementary instrumentation will provide all sky coverage for $X-$ and gamma ray bursts. An articulated platform with its own star tracker will perform independent observations with X-ray and EUV telescopes. The satellite will be launched into a deep, highly eccentric, orbit with a period which could be as long as four days and from which long duration observations can be made.

\section{XSPECT TELESCOPE SYSTEM}

A brief description of the X-ray optics, the focal plane and other instrumentation is contained in the sections which follow.

\section{$2.1 \mathrm{X}$-ray Optics}

Specular reflection of $\mathrm{X}$-rays is possible only at small incident angles under conditions of total external reflection. The critical angle, beyond which reflection does not occur, depends upon the density of electrons in the reflector and the energy of the incident $X$-ray. These requirements have led to the design of $X$-ray imaging systems which have two reflectors in the form of surfaces of revolution proposed by Wolter 


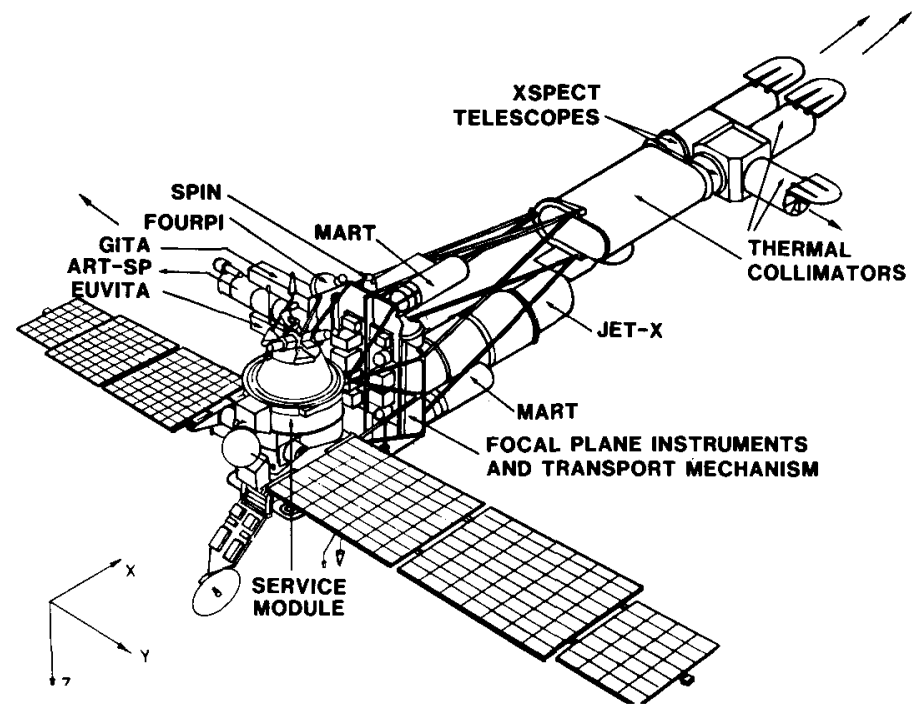

Figure 1. SPECTRUM RÖNTGEN GAMMA satellite. (R.S. Kremnev, Babakin Center)

(1952 a,b). The best use of the available telescope aperture is obtained by nesting as many pairs of reflectors as possible but care must be taken to minimize the deviation fron the condition of exact alignment and distortions from the exact shell figure.

Roughness, both short and long range, on scales as small as atomic sizes will introduce scattering halos around the geometrically perfect image of a point source. The angular diameter of the circle which encloses half of the photons in the image of a point source is defined as the Half Power Width (HPW) and is very useful in determining the performance of the system. It is usually referred to when questions of source confusion arise. For a recent review of these topics see Aschenbach (1985).

If the length of a conical surface is made sufficiently short, then the deviation from the ideal paraboidal and hyperboloidal Wolter I surfaces can be made extremely small. Ray tracing studies have shown that the contribution to the HPW from these deviations can be held to $<20$ arcsec. A quite large fraction of the aperture can be filled by densly nesting many shells of thin foils. This approach not only produces a very large collecting area, but also extends the energy range of the telescope to perhaps $20 \mathrm{keV}$, or more, since shells with very shallow grazing angles can be placed close to the optical axis.

The design goal for the XSPECT telescope HPW is $<2$ arcmin and the difference between this value and the contribution from the conical geometry represents the combined error budget which is allowed for all the manufacturing tolerances. Our approach, which follows the original suggestion by Serlemitsos, Petri, Glasser, and Birsa (1984), is to manufacture the telescope in two sections joined together by a flange which also serves as the interface with the optical bench. Each half is divided into independent 


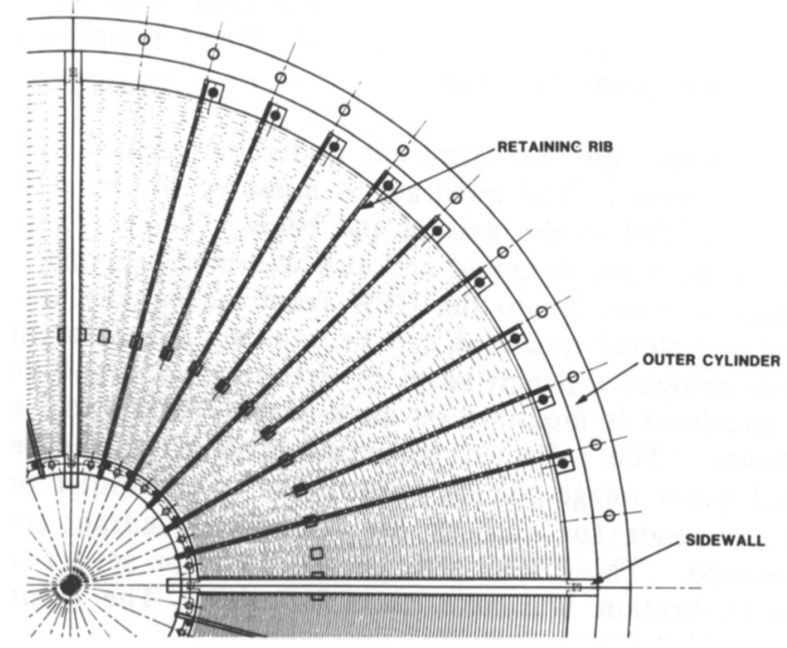

Figure 2. One quadrant of the XSPECT telescope. (J. Polny, DSRI)

quadrants which are keyed to a central reference hub (Figure 2). Foils are fixed into grooves in the quadrant side plates and held in place by adjustable radial spokes. Critical manufacturing steps are carried out in numerically-controlled, spark-cutting machines under tight temperature control.

Commercially available aluminum foils with thicknesses between 0.1 and $0.3 \mathrm{~mm}$ can be obtained with relatively smooth surfaces. Samples are evaluated by measuring the scattering of light from a $\mathrm{He}-\mathrm{Ne}$ laser which samples the long range surface structure in the surface of the foil. Accepable material is cut to the appropriate shape and rolled to approximately the correct figure. Next, the foils are coated with a thin layer of acrylic resin which tends to smooth out the short range residual graininess in the surface. Finally, a layer of gold (or perhaps other materials under study) is evaporated to form the high density reflecting surface. Surfaces prepared in this way can have a surface roughness on the order of 3 A RMS which is smooth enough to remove totally the effects of small angle $X$-ray scattering. The long rang structure inherent in rolled aluminum foils is not removed and can contribute $\sim 1$ arcmin or more to the HPW (Kunieda and Serlemitsos, 1988).

Materials other than aluminum foils are being investigated by various groups. Tanaka and Makino (1988) report on plastic foils which are cut, rolled and glued to form the complete conical surface and are then assembled into the structure after gold evaporation. Laboratory tests are very impressive, but in flight thermal effects which could cause figure altering gradients may pose practical limitations. More promising is the possibility of using galvano-plastically deposited nickel foils (Hudec and Valnicek, 1985) either as full cones or as rolled foils. Laser scattering tests at DSRI of foils produced on ordinary window glass indicate very smooth surfaces since almost no scattering is observed. The foils are $20 \times 30 \mathrm{~cm}$ in size and a large area in the central portion has a thickness variation of no greater than $1.6 \%$ (Hudec, 1988). The foils lie flat, an indication of relatively small internal stresses and a property which becomes important in 
insuring the proper figure of the rolled foil. The long range waves in the glass surface are, however, faithfully reproduced in the foil. Super-polished glass substrates are being prepared for the next production run.

Foils are mounted into the quadrant under the control of a well collimated optical beam $30 \mathrm{~cm}$ in diameter. The quadrant is mounted to the reference hub whose axis is made accurately parallel to the axis of the beam. This proceess allows individual foils to be evaluated as they are mounted and also will measure, apart from optical diffraction effects, the total contribution to the HPW from mechanical defects in the foils and the structure. The completed quadrant can be evaluated in a facility, now under construction, which will provide a pencil beam of extremely well collimated and monochromatic $X$-rays. The quadrant is mounted on an $x-y$ table which can be used to make a raster scan of the beam. The reflected beam is registered by an imaging detector and the synthesized full beam image can be constructed by computer processing. Similar measurements can be made for each of the full four quadrant sections and for the fully assembled telescope. These tests will be repeated for several energies. It is expected that the final calibration will take place in orbit. The laboratory measurements are used only to control the manufacturing processes.

The parameters being considered for the baseline design are listed in Table 1 .

Table 1. XSPECT Conical Approximation to Wolter 1 Optics

$\begin{array}{lrl}\text { Focal length } & 8 & \mathrm{~m} \\ \text { Number of shells } & \text { ca. } 118 & \\ \text { Outer shell diameter } & 60 & \mathrm{~cm} \\ \text { Inner shell diameter } & 16 \mathrm{~cm} \\ \text { Shell length } & 20 & \mathrm{~cm} \\ \text { Shell thickness } & 0.3 & \mathrm{~mm} \\ \text { Minimum shell separation } & 0.8 & \mathrm{~mm} \\ \text { Total reflecting surface } & 65 \mathrm{~m}^{2} \\ \text { Mass per telescope } & 50 & \mathrm{~kg} \\ \text { Field of view (FOV) } & 60 & \mathrm{arcmin} \\ \text { Half power width (HPW) } & <2 & \text { arcmin } \\ \text { Shell material } & & \text { aluminum (nickel) } \\ \text { Reflecting material } & & \text { gold (iridium) } \\ \text { Effective area (one module, } & 1700 & \mathrm{~cm}^{2} @ 2 \mathrm{keV} \\ \quad \text { without detector efficiency) } & 1200 & 8 \\ & 60 & 20\end{array}$

Figure 3 compares the collecting area vs. energy which can be expected for a single XSPECT telescope with that of several other past or future missions. Measurements of reflection efficiencies for gold at several energies have been made at DSRI and have been found to agree well with the theoretical values which have been used to construct the XSPECT curve.

\section{$2.2 \mathrm{X}$-ray Detectors}

A detector with a minimum diameter of $15 \mathrm{~cm}$ will match the focal plane scale of $2.3 \mathrm{~mm} / \mathrm{arcmin}$ and a field of view (FOV) of 1 degree. The resolution of the detector should be fine enough to adequately sample the $4.6 \mathrm{~mm}$ diameter pixel which is equivalent 


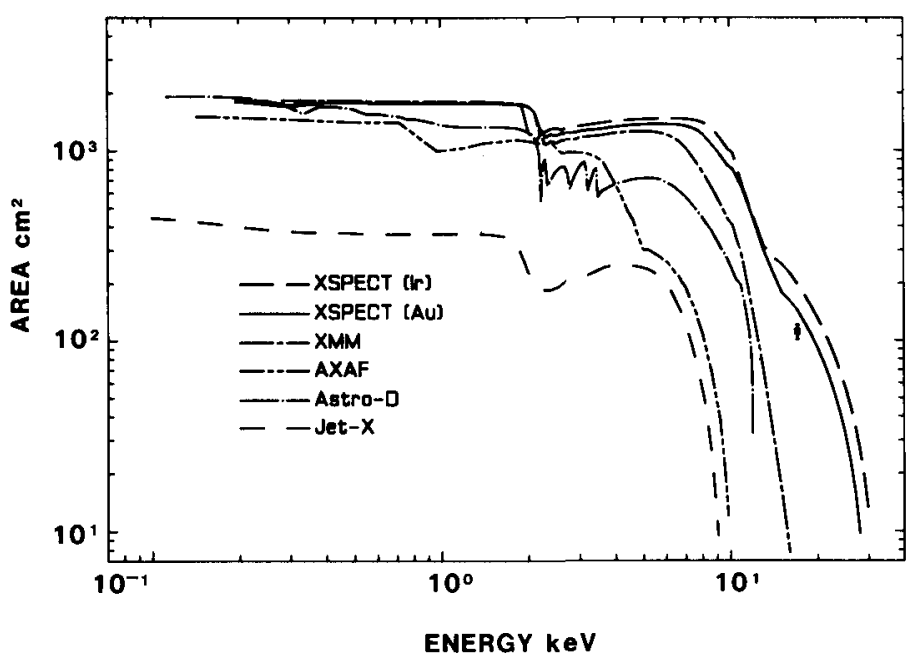

Figure 3. On axis effective area v.s. energy for various telescopes (single module, without detector efficiency). (N.-J. Westergaard, DSRI)

to the HPW (and allow for some improvement). The final requirement is that the detector span the entire range of energies reflected from the telescope with an energy resolution which will allow significant astrophysical interpretation of the data. From the wide variety of gas-filled and solid-state detectors, a large area position sensitive proportional counter and a small array of silicon solid state detectors have been chosen to meet the scientific requirements of the mission. Other detectors are under consideration but they are not discussed here.

2.2.1 Position sensitive proportional counter (PSPC). Detectors of this type have been flown successfully on EINSTEIN, unsuccessfully on EXOSAT and are to be flown on ROSAT. They offer the advantage of well established technology and can be made to cover large areas. All gas filled detectors must have an entrance window and the strong variation of $\mathrm{X}$-ray attenuation in typical window materials, particularly at energies $<2$ $\mathrm{keV}$, makes it difficult to cover the very broad XSPECT energy range in a single detector. The baseline detector system consists of two independent detectors, one for low energy which has a thin stretched plastic window and a gas flow system to replenish leakage and the other for high energy which has a sealed beryllium window. The characteristics of these detectors are summarized in Table 2.

Table 2. XSPECT Detector Specifications

Low Energy High Energy

$\begin{array}{lll}\text { Diameter of active area } & 15 \mathrm{~cm} & 15 \mathrm{~cm} \\ \text { Energy range } & 0.5-3.0 \mathrm{keV} & 2-20 \mathrm{keV} \\ \text { Energy resolution } & 25 \% \text { at } 2 \mathrm{keV} & 13 \% \text { at } 6 \mathrm{keV} \\ \text { Position resolution (x and y) } & 2 \mathrm{~mm} & 1 \mathrm{~mm} \\ \text { Quantum efficiency } & >0.7 ; \mathrm{E}>0.8 \mathrm{keV} & >0.7 ; 2<\mathrm{E}<10 \mathrm{keV}\end{array}$


At present, multiwire (MWPC), microstrip (MSPC) and parallel gap (PGPC) proportional counters are under development at DSRI (Budtz-Jørgensen, et al, 1989). Of these, the first has been chosen as the baseline even though it does not meet the (selfimposed) energy resolution requirements specified in Table 1 . The details of this detector have been given elsewhere (Madsen, et al, 1985). Only two layers of wires are used; an anode plane with $12 \mu \mathrm{m} \mathrm{NiCr}$ wires placed $2 \mathrm{~mm}$ apart and a field-controlling cathode plane with wires also spaced $2 \mathrm{~mm}$ apart located $1.5 \mathrm{~mm}$ above and parallel with the anode plane. The wires are supported at each end on insulating bars which positions each wire to within $\pm 2 \mu \mathrm{m}$.

A cathode plane divided into four segments is located at a suitable distance below the anode plane. Each segment is divided into strips which are interleaved with those of the other segments. Primary electrons created in the initial X-ray absorption event drift towards the anode wires in the relatively weak field between the entrance window and the cathode wires. An avalanche occurs in the strong field surrounding the anode and the movement of ions and electrons induces charges of opposite polarity on the anodes and cathodes. The geometry allows more than $50 \%$ of the charge produced in the avalanche to be induced on the cathode plane. The variation in strip geometry allows the centroid position of the charge cloud to be calculated from the charge signals collected on the four segments.

The dominant contribution to the detector resolution is noise in the cathode plane preamplifier and it is, therefore, important to reduce the interelectrode capacitance on the cathode plane and to use low noise preamplifiers (less than $300 \mathrm{rms}$ electrons). Results obtained from a breadboard detector filled with a mixture of $\mathrm{Ar}+10 \% \mathrm{CH}$ (P10) gave position resolutions of 0.42 and $0.34 \mathrm{~mm}$ (FWHM) and energy resolutions of $40 \%$ and $22 \%$ (FWHM)for 1.5 and $5.9 \mathrm{keV}$ X-rays, respectively. Pulse height and rise time discrimination together with inter-electrode voltage comparison yield a combined background rejection efficiency of $>99 \%$ and an acceptance efficiency of $71 \%$ at $5.9 \mathrm{keV}$ (Madsen, et al, 1985).

Limitations imposed by having suspended wire anode and cathode planes restrict the achievable energy resolution. It is difficult to insure that the wires stay parallel in the applied electric field and non-uniform gas amplification is a commonly seen, particularly in large area detectors. A novel approach, in which the wires are replaced by very narrowly spaced conducting "microstrips (MS)" accurately deposited $( \pm 2 \mu \mathrm{m})$ on an insulating substrate, has made it possible to circumvent this problem (Oed,1988). The wire planes in the DSRI breadboard detector were replaced by a MS which was supplied by Oed. A gas gain of $10^{3}$ was obtained at a voltage of $600 \mathrm{~V}$ and energy resolutions of $27.4 \%$ and $13.4 \%$ were obtained with $\mathrm{Pl} 0$ gas for 1.5 and $5.9 \mathrm{keV} \mathrm{X}$-rays, respectively. Similar resolutions were obtained with propane gas (Budtz-Jørgensen, et al, 1989). Tests with new thin large area MS plates which are designed to induce strong signals on the cathode segments are in progress. Various readout schemes are also under study.

Ultra-thin, strong window materials which are nearly leak-proof have recently become available (Sipilä, 1988). At present they are being produced with relativly small areas but it is expected that the requirements for the XSPECT detectors can be met in the near future. The window transmission is equivalent to $0.5-1.0 \mu \mathrm{m}$ of stretched polypropylene. The ideal solution for XSPECT is to combine both the high- and low energy detectors into a single unit which has an ultra thin window and a MS plate and, thereby, fulfills the requirements specified in Table 2. 
2.2.2 Solid state spectrometer array (SSSA). The significant improvement in energy resolution obtained from these devices more than compensates for the requirement that the detector be cooled. Solid state $\mathrm{X}$-ray detectors are usually made from lithium-drifted-silicon [Si(Li)]. The primary difference from a gas detector is that, although much less energy is required to liberate an electron within the silicon, there is no equivalent to gas amplification. The primary charge must be detected by extremely low noise, cooled preamplifiers which can contribute $\sim 100 \mathrm{eV}$ to the FWHM and this is the limiting resolution for $X$-rays with energies below $\sim 2 \mathrm{keV}$. At $6 \mathrm{keV}$, a resolution of $150 \mathrm{eV}$ $(E / \Delta E=40)$ is common, but recent preamplifier improvements have lowered this value to $130 \mathrm{eV}(\mathrm{E} / \Delta \mathrm{E}=46)$ with expectations of $50 \mathrm{eV}(\mathrm{E} / \Delta \mathrm{E}=10)$ at $500 \mathrm{eV}$.

The combination of layers of gold which acts as the charge collector and of nonactive ("dead") silicon on the front surface of the detector limits the low energy response of the detector while the thichness (usually 2-4 mm) limits the high energy response. Filters to reduce infrared, visible and ultraviolet background also limit the low energy response. Imaging detectors can be either arrays of discrete elements or segmented blocks within a large single crystal. In either case it would be difficult to match the pixel size requirements of a high resolution telescope such as AXAF, but the needs of XSPECT do not pose a practical limitation.

The deep orbit makes it possible to use a radiative cooler to bring the detector system to the proper operating temperature. The number of pixels will be limited be the minimum power required by the preamplifier and the heat leaks between the detector assembly and the heat radiator. The minimum design is 7-9 pixels with 4-6 mm diameter, but the number could increase when ongoing development work is evaluated.

\subsection{Objective Crystal Spectrometer (OXS)}

Bragg crystal instruments can provide very high energy resolution but with low efficiency since the multiplex advantage inherent in broad band and grating spectrometers is lost because of the restrictive nature of the $\mathrm{X}$-ray diffraction process. In addition, their high dispersion insures that the effects of telescope blur do not dominate the energy resolution and it is, therefore, possible to design instruments which will operate successfully in conjunction with low resolution telescopes. Substructure within the helium like emission lines including the diagnostically important satellite lines can be resolved for all of the cosmically abundant species and, in some cases, the structure of individual lines can be studied. Many important spectral lines are well separated in energy and bright sources can be imaged at each energy without the confusion of overlapping common for grating instruments.

The approach chosen for the XSPECT spectrometer is to separate the spectroscopic and imaging functions of the instrument. A large, flat, free-standing crystal would be a fine spectrometer for a bright point source or, with suitable mechanical collimation, for an extended source. Excellent solar spectra have been obtained this way. For the relatively weak cosmic $X$-ray sources the background introduced from the necessarily large active detector volume overwhelms the signal. With a telescope following the crystal (Figure 4) the background attributable to the detecting pixel volume shrinks dramatically and extended sources can be imaged as well (Schnopper and Byrnak, 1987). The angular range for which a crystal reflects a monochromatic beam is typically $0.5-$ 1.0 arcmin and, therefore, this concept is ideally suited for XSPECT. 


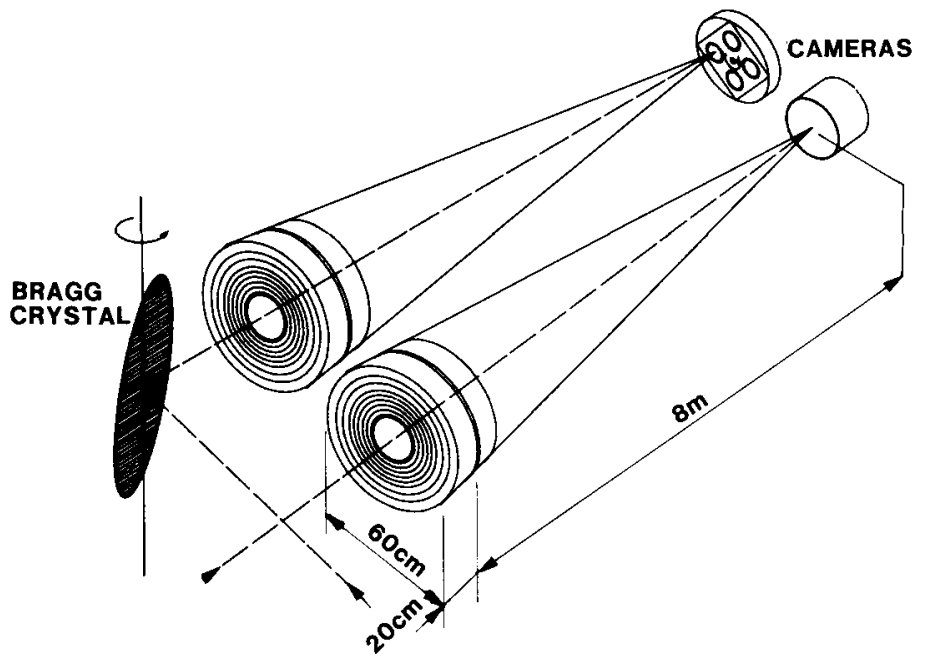

Figure 4. XSPECT spectrometer, telescope and detectors.

\subsection{The XSPECT Telescope Assembly}

XSPECT is illustrated in Figures 1 and 4 and consists of the telescope modules; the Bragg crystal panel; the complete complement of focal plane instruments; the structural, thermal and focal plane assemblies; and the star tracker. The telescope is launched in a folded configuration which allows the satellite to fit within the shroud of the PROTON launcher and is deployed when orbital altitude is reached. Babakin Center is responsible for the structure and deployment mechanism, the thermal collimators (which keep the telescopes within the allowed temperature range and the focal plane transport mechanism. The Institute of Electromechanics of the USSR Academy of Sciences is responsible for the thermal radiator.

\section{SCIENTIFIC PROGRAM}

The large collecting area and broad energy range provided by the telescope and the deep orbit of the satellite will lead to a comprehensive observing program. With low resolution telescopes such as XSPECT, source confusion can become a limiting factor in the performance of the telescope. The slope of the relationship between the number of sources $N(>S)$ with flux greater than $S$ together with the spectral shape of a typical source determine when a particular telescope system becomes confused. Sky surveys already carried out are consistent with a slope of -1.5 for the $\log N(>S)-\log S$ curve, but recent work by Hamilton and Helfand (1987) shows that fluctuations in the pixel to pixel background data indicate a flattening of the curve for flux levels below the EINSTEIN deep survey limit $\left[S_{0}=2.6 \times 10^{-14} \mathrm{erg} \mathrm{cm}-2 \mathrm{~s}^{-1}(1-3 \mathrm{keV})\right.$ and $4.0 \times 10^{-14} \mathrm{erg} \mathrm{cm}^{-2} \mathrm{~s}^{-1}(3-10$ kev)]. The implications are that for $S<S_{0}$ there can be many more faint objects than expected from extrapolation of surveys for which $S>S_{0}$ and that these faint sources will not be detected with the most sensitive telescopes now planned. 


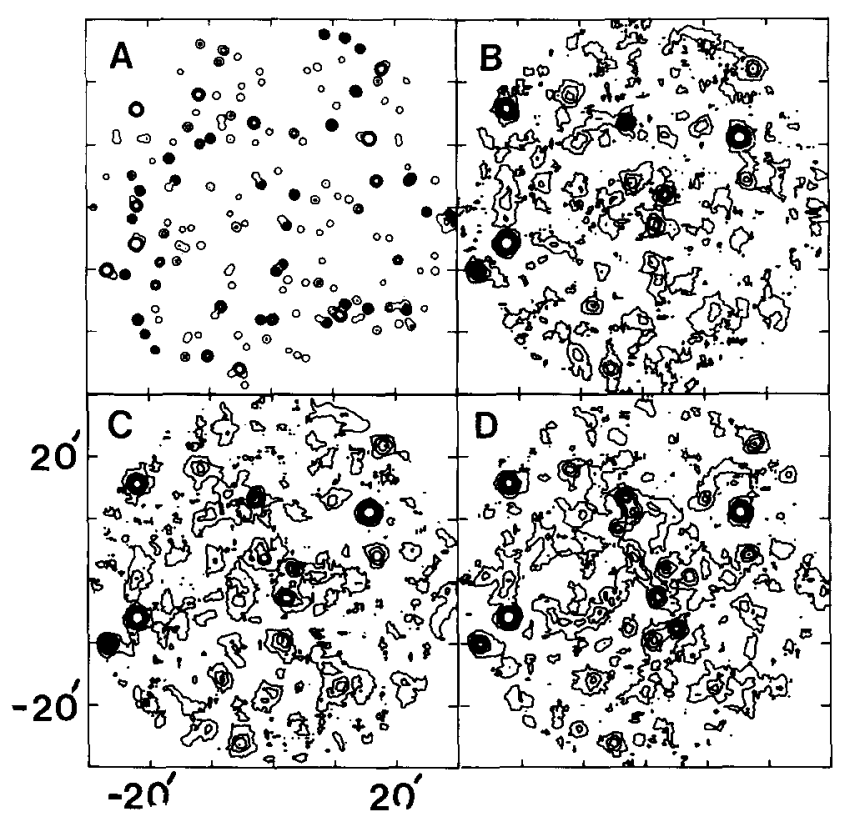

Figure 5. Simulated response of the PSPC for $10^{5} \mathrm{~s}$ exposures for sources distributed according to $\log N(>S)-\log S$ with various slopes. a) The input catalog; b), c) and d) Slopes of 1.05, 1.20 and 1.50. (N.-J. Westergaard, DSRI)

Simulations for observations of $10^{5} \mathrm{~s}$ have been made under various assumptions for the slope of the $\log (\mathrm{N}>\mathrm{S})-\log S$ curve. These are shown in Figure 5 . The point response function of the telescope has been modeled to included construction errors and scattering up to a HPW of $2 \operatorname{arcmin}^{2}$. Sources with spectra similar to the X-ray background are assumed. The results indicate that sources with fluxes well below $S_{0}$ can be detected if the distribution is truely flattened. Knowledge of precise source positions to be obtained from the ROSAT catalog will be invaluable in sorting out the problems arising from confused regions. Assuming a $4 \operatorname{arcmin}^{2}$ resolution and requiring that there be 40 beam areas surrounding each source there are 22.5 resolved bins $/ \mathrm{deg}^{2}$ or

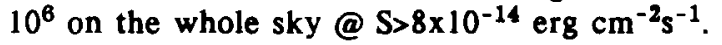

A few examples illustrate the sensitivity of the instrument. The Seyfert galaxy NGC4151 $(d=20 \mathrm{Mpc}, \mathrm{z}=0.004)$ with $S \simeq 10^{-10} \mathrm{erg} \mathrm{cm}^{-2} \mathrm{~s}^{-1}(2-10 \mathrm{keV})$ could be detected

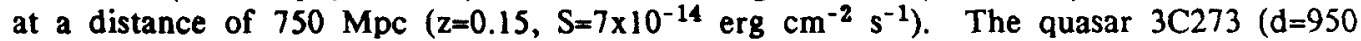
Mpc, $z=0.2$ ) with $S=6 \times 10^{-11}$ erg cm $\mathrm{cm}^{-2} \mathrm{~s}^{-1}$ could be detected at a redshift for which evolution is an important factor. The Coma Cluster $(\mathrm{d}=113 \mathrm{Mpc}, \mathrm{z}=0.02)$ with $\mathrm{S}=3.2 \mathrm{x}$

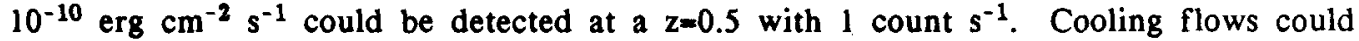
be detected at $z=0.3$. These detections include physically significant measurements of spectral index or temperature.

The large collecting area combined with the broad energy range will allow studies of the surface brightness distribution of clusters at large distances from the center and, 
if they exist, measurements of the surface brightness distribution of superclusters. Dark matter would contribute to both of these distributions.

A $10^{5} \mathrm{~s}$ observation binned in $10 \operatorname{arcmin}^{2}$ bins will include $\sim 140$ and $<50$ counts of signal and noise, respectively. The gain uniformity in the gas detector will permit precise studies of the bin-to-bin fluctuations to be made which will reveal the spectrum and luminosity function of the unresolved portion of the $X$-ray background. These studies should also discover 1 quasar every $10^{4} \mathrm{~s}$ or $10^{3}$ sources/year with $25 \%$ observing ef ficiency.

Provided that they are not confused, bright $x$-ray sources in nearby galaxies, i.e. a CYG X-1 in M31 and SN1987A in the LMC, are easily detected. M31 is a good example since all the EINSTEIN sources with the exception of those at the very center of the galaxy could be studied in great detail.

Within the Galaxy there are many topics to chose from where the XSPECT system can be expected to provide excellent data. Of particular importance are sub-millisecond studies of time variability, spectra from all classes of stars, plasma diagnostics of the interstellar medium, diagnostics of accretion discs, and high- and low resolution temperature and density mapping of supernova remnants. Emission lines from compact objects which can be broadened by various scattering processes can be detected over the broad XSPECT energy range as can low energy cyclotron emission- and/or absorption features.

\section{ACKNOWLEDGEMENTS}

The staff at DSRI including: B.P. Byrnak, F.E. Christensen, A. Hornstrup, P. Jonasson, M.M. Madsen, H.U. Nørgaard-Nielsen and N.-J. Westergaard have made significant contributions to the work reported here. I thank them warmly for their assistance in preparing the manuscript.

\section{REFERENCES}

Aschenbach, B. 1985, Rep. Prog. Phys., 48, 579-629.

Budtz-Jørgensen C., Madsen, M.M., Jonasson,P., Schnopper, H.W., and Oed, A. 1989, SPIE Proc., 982, in press.

Hamilton, T.T., and Helfand, D.J. 1987, Ap. J., 318, 93-102.

Hudec, R., and Valnicek, B. 1985, SPIE Proc., 597, 111-118.

Hudec, R. 1988, private communication.

Kunieda., and Serlemitsos, P.J. 1988, SPIE Proc., 830, 12-15.

Madsen, M.M., Jonasson, P., Jensen, P.L., Rasmussen, H.E., Ørup, P., and Schnopper, H.W. 1985, SPIE Proc., 597, 199-205.

Oed, A. 1988, Nucl. Instr. Meth., A263, 351-359.

Schnopper, H.W., and Brynak, B.P. 1987, Appl. Opt., 26, 2871-2876.

Serlemitsos, P.J., Petre, R., Glasser, C., and Birsa, F. 1984, IEEE Trans. Nuc. Sci., NS31, 786-790.

Sipilă, H. 1988, private communication.

Tanaka, Y.,and Makino, F., 1988, SPIE Proc., 830, 242-244.

Wolter, H. 1952a, Ann. Phys., 10, 94.

1952b, Ann. Phys., 10, 286. 


\section{DISCUSSION-H. Schnopper}

S. Kahn: Why are you using a single crystal plane rather than a venetian blind approach, which would take less room in front of the mirror?

$H$. Schnopper: A venetian blind would only be effective for shallow incident angles, i.e. for reflection gratings. At $\sim 45^{\circ}$ incident angle the blind elements would occult each other; a single plane is the best geometry.

B. Smith: Did you mention the wavelengths your crystals will cover?

H. Schnopper: We plan to cover the oxygen, silicon, sulfur and iron K energy bands.

P. DeKorte: What will be the spectral resolution of the Bragg Spectrometer taking into account the system resolution and the spacecraft attitude uncertainties.

H. Schnopper: We expect an $\mathrm{E} / \Delta \mathrm{E}$ in the range $5 \times 10^{2}-2 \times 10^{3}$, with the best performance at iron. 